\title{
Efficacy and Safety of Dupilumab in Clinical Practice: One Year of Experience on 165 Adult Patients from a Tertiary Referral Centre
}

\author{
Maddalena Napolitano (D) - Maria Ferrillo - Cataldo Patruno • \\ Massimiliano Scalvenzi · Mirella D'Andrea · Gabriella Fabbrocini
}

Received: December 30, 2020 / Published online: March 13, 2021

(C) The Author(s) 2021

\section{ABSTRACT}

We have read with great interest the article by Kreeshan et al., which reported data on effectiveness and laboratory safety of dupilumab. We performed a retrospective study including 165 adult patients affected by moderate-to-severe atopic dermatitis (AD) and treated with dupilumab for at least 52 weeks. A significant improvement in eczema area severity index (EASI) score after 16 and 52 weeks of treatment with dupilumab was observed. The mean EASI

This comment refers to the article available online at https://doi.org/10.1007/s13555-020-00469-6.

M. Napolitano $(\bowtie)$

Department of Medicine and Health Sciences

Vincenzo Tiberio, University of Molise,

Campobasso, Italy

e-mail: maddy.napolitano@gmail.com

M. Ferrillo · M. Scalvenzi · M. D’Andrea ·

G. Fabbrocini

Section of Dermatology, Department of Clinical

Medicine and Surgery, University of Naples Federico

II, Naples, Italy

C. Patruno

Department of Health Sciences, University of

Catanzaro "Magna Graecia”, Catanzaro, Italy score at baseline was $28.84 \pm 6.4$ and significantly reduced to $10.05 \pm 8.00$ at 16 weeks $(p<0.001)$, and to $3.04 \pm 4.73$ at 52 weeks $(p<0.001)$, with a mean percentage reduction of $65.15 \%$ and $89.45 \%$, respectively. Efficacy of dupilumab was demonstrated by a significant reduction of all the scores (P-NRS, S-NRS and DLQI). Furthermore, no patient discontinued the drug because of inefficacy. Fifty-seven out of 165 (34.54\%) patients reported at least one adverse event (AE) during the 52-week treatment. Our study confirms that dupilumab can represent a long-term treatment for moderateto-severe adult $\mathrm{AD}$, beyond 16 weeks. In our experience, dupilumab demonstrated a favourable safety profile at 52 weeks and only a few patients had to discontinue the treatment because of AEs.

Keywords: Atopic dermatitis; Daily practice; Dupilumab; Eczema; Severity 


\section{Key Summary Points}

Dupilumab, the first biologic drug approved for $\mathrm{AD}$ treatment, has been available since 2018 .

The efficacy and safety were demonstrated in clinical trials. Currently, data regarding its daily practice setting, real-word effectiveness and tolerability up to 52 weeks are limited.

This study presents a real-life experience on dupilumab effectiveness, safety and tolerability in a cohort of 165 patients treated for 52 weeks.

The study shows that the long-term treatment with dupilumab in daily practice is effective and safe for patients with moderate-to-severe atopic dermatitis.

\section{DIGITAL FEATURES}

This article is published with digital features, including a summary slide, to facilitate understanding of the article. To view digital features for this article go to https://doi.org/10.6084/ m9.figshare.13691539.

\section{COMMENTARY}

We have read with great interest the article by Kreeshan et al., which reported data on effectiveness and laboratory safety of dupilumab in a real-word cohort of 164 patients affected by atopic dermatitis (AD) followed over 12-30 weeks [1]. The authors reported that $58.96 \%$ of patients achieved at least a 75\% improvement in eczema area severity index (EASI75), while $37.31 \%$ achieved EASI90 at $12-16$ weeks. Regarding laboratory test, eosinophilia started after initiation of dupilumab in $24 \%$ of cases, but it caused no interruption of treatment.

We performed a retrospective study including adult patients affected by moderate-to- severe $\mathrm{AD}$ and treated with dupilumab for at least 52 weeks. The study was conducted at the Atopic Unit of University of Naples Federico II from June 2018. For each patient the following information was collected: demographic and clinical data (age, sex, medical history, clinical phenotype of $\mathrm{AD}$ ), comorbidities (atopic and non-atopic), concomitant medications or procedures, adverse events (AEs), efficacy outcomes to previous treatments. Total serum immunoglobulin (Ig)E and eosinophil count, EASI, Numerical Rating Scale (NRS) for pruritus (P-NRS) and sleep (NRS-S) and Dermatology Life Quality Index (DLQI) were assessed at baseline, week 16 and week 52. Descriptive statistics were calculated for each demographic and clinical variable, using frequencies and percentage for categorical variables and mean \pm standard deviation (SD) for continuous ones. GraphPad Prism software (v.4.0; GraphPad Software Inc. La Jolla, CA, USA) was used for all statistical analyses. A value of $p<0.05$ was considered significant.

All procedures performed in studies involving human participants were in accordance with the ethical standards of the institutional and/or national research committee and with the 1964 Helsinki Declaration and its later amendments or comparable ethical standards. The study has been approved by ethics committee of University of Naples Federico II and informed consent was obtained from all individual participants involved in the study.

From June 2018, 325 (155 male, 170 female; age $45.29 \pm 19.11$ years) patients started treatment with dupilumab, but of these a total of 165 patients [95 men (57.57\%); mean age $43.35 \pm 18.96$ years (range 18-90)] was enrolled because they achieved 52 weeks of treatment (Table 1). AD had developed before the age of 18 (persistent AD) in 94/165 (56.96\%) patients, whereas in 71/165 (43.04\%), the onset of $\mathrm{AD}$ was directly in adulthood (adult-onset AD). The most frequent $\mathrm{AD}$ phenotype was flexural dermatitis alone, observed in 86/165 (52.19\%) patients, followed by prurigo nodularis $(27 / 165$; $16.73 \%)$, head/neck eczema 21/165 (16.73\%), generalized eczema $(13 / 165 ; 7.75 \%)$, hand eczema 20/165 (12.24\%), nummular eczema $(13 / 165 ; 8.16 \%)$ and erythroderma $(2 / 165$; 
Table 1 Clinical characteristics of 165 patients with atopic dermatitis and treated with dupilumab

\begin{tabular}{|c|c|}
\hline Variable & $\begin{array}{l}\text { Value } \\
n(\%)\end{array}$ \\
\hline Age (years) & $43.35 \pm 18.96$ \\
\hline Sex, female & $70(42.42)$ \\
\hline \multicolumn{2}{|l|}{$\mathrm{AD}$ pattern } \\
\hline Persistent & $94 / 165(56.96)$ \\
\hline Late onset ( $\geq 18$ years) & $71 / 165(43.04)$ \\
\hline \multicolumn{2}{|l|}{ Clinical scores at baseline } \\
\hline EASI score & $28.84 \pm 6.4$ \\
\hline Peak score on NRS for pruritus & $8.63 \pm 1.67$ \\
\hline Peak score on NRS for sleep & $6.42 \pm 3.15$ \\
\hline DLQI & $22.93 \pm 6.24$ \\
\hline \multicolumn{2}{|l|}{ Atopic comorbidities } \\
\hline Rhinitis & $36 / 165(21.81)$ \\
\hline Asthma & $22 / 165(13.33)$ \\
\hline Conjunctivitis & $18 / 165(10.90)$ \\
\hline Food allergy & $6 / 165(3.63)$ \\
\hline \multicolumn{2}{|l|}{ Non-atopic comorbidities } \\
\hline $\begin{array}{l}\text { Hypertension and cardiovascular } \\
\text { disorders }\end{array}$ & $24 / 165(14.54)$ \\
\hline Hyperlipidaemia & $20 / 165(12.12)$ \\
\hline Psychiatric/psychological disorders & $11 / 165(6.66)$ \\
\hline Diabetes & $6 / 165(3.63)$ \\
\hline Obesity & $2 / 165(1.21)$ \\
\hline \multicolumn{2}{|l|}{ Previous systemic treatments for $\mathrm{AD}$} \\
\hline Cyclosporine A (CsA) & $\begin{array}{l}109 / \\
165(66.06)\end{array}$ \\
\hline Systemic corticosteroids & $\begin{array}{l}126 / 165 \\
(76.36)\end{array}$ \\
\hline Phototherapy & $62 / 165(37.57)$ \\
\hline Methotrexate & $4 / 164(2.42)$ \\
\hline Azathioprine & $1 / 165(0.60)$ \\
\hline
\end{tabular}

$1.21 \%)$. The coexistence of more than one phenotype was found in 25/165 (15.15\%) patients. The main associations were flexural dermatitis with head/neck eczema or hand eczema in 7 (4.24\%) and 8 (4.84\%) patients, respectively. No statistically significant differences were found regarding onset $\mathrm{AD}$ and different phenotype.

Fifty-two out of 165 (31.51\%) patients had one or more allergic comorbidities. The most frequent reported allergic disorders were rhinitis $(36 / 165 ; 21.81 \%)$, asthma $(22 / 165 ; 13.33 \%)$, conjunctivitis $(18 / 165 ; 10.90 \%)$ and food allergy $(6 / 165 ; 3.63 \%)$. Regarding the main previous therapies, $66.06 \%(109 / 165)$ of the patients had received CsA, and $76.36 \%(126 / 165)$ had received systemic corticosteroids, $37.57 \%$ (62/ 165 ) had been treated with phototherapy (narrow-band UVB).

Eosinophilia ( $>500$ eosinophils $/ \mathrm{mm}^{3}$ ) was detected in $8.25 \%(20 / 165)$ of patients at baseline and in $21.21 \%(35 / 165)$ at week 16 $(p<0.05)$. At baseline, total IgE levels were above the normal range $\left(0-150 \times 10^{3} \mathrm{IU} / \mathrm{L}\right)$ in $79 / 165(47.87 \%)$ patients. In these subjects the mean value of $2532 \times 10^{3} \mathrm{IU} / \mathrm{L}$ and decreased in $44 / 79(26.66 \%)$ patients to a mean value of $1119 \times 10^{3} \mathrm{IU} / \mathrm{L}$ at week $16(p=0.6)$.

A significant improvement in EASI score after 16 and 52 weeks of treatment with dupilumab was observed (Figs. 1, 2). The mean EASI score at baseline was $28.84 \pm 6.4$ and significantly reduced to $10.05 \pm 8.00$ at 16 weeks $(p<0.001)$, and to $3.04 \pm 4.73$ at 52 weeks $(p<0.001)$, with a mean percentage reduction of $65.15 \%$ and $89.45 \%$, respectively. In our experience, the mean EASI value at baseline was higher than the one reported by the authors because, according to the Italian Medical Agency criteria, dupilumab can be prescribed to patients with EASI $\geq 24$ at baseline and/or with contraindication, side effects or failure to cyclosporine. We observed that 20/165 $(12.12 \%)$ patients achieved EASI50 $(\geq 50 \%$ reduction in EASI score from baseline to week 52), 68.48\% (113/165) achieved EASI75 $(\geq 75 \%$ reduction in EASI score from baseline to week 52), and 7.27\% (12/165) achieved EASI90. Likewise, it has been observed a significant improvement in both pruritus and sleep numerical rating score (P-NRS and S-NRS) and DLQI from baseline to all time points (Fig. 1). 
P-NRS had a mean value of $8.63 \pm 1.67$ at baseline vs $4.32 \pm 2.10$ and $1.40 \pm 2.12$ at 16 and 52 weeks, respectively. The mean S-NRS also showed a significant reduction from baseline to both time points $[6.42 \pm 3.15$ at baseline vs $3.55 \pm 2.40$ and $0.33 \pm 0.92$ at 16 and 52 weeks, respectively; $(p<0.01)$ ]. As for quality of life, DLQI score at baseline was $22.93 \pm 6.24$ vs $7.01 \pm 5.41$ and $2.25 \pm 3.88$ at 16 and 52 weeks, respectively $(p<0.01)$. No statistically significant differences were found between $\mathrm{AD}$ phenotypes to treatment response.

Fifty-seven out of 165 (34.54\%) patients reported at least one $\mathrm{AE}$ during the 52 -week treatment. Overall, treatment-emergent AEs consisted of 16 cases $(9.69 \%)$ of mild conjunctivitis treated with tear substitutes and corticosteroid eye drops. Three of 16 patients had a history of conjunctivitis before dupilumab therapy. In two patients $(1.21 \%)$, severe conjunctivitis caused treatment interruption. Other common AEs were injection-site reaction (11/ $165 ; 6.66 \%)$, headache $(9 / 165 ; 5.45 \%)$, fatigue $(7 / 165 ; 4.24 \%)$, psoriasiform eruption $(5 / 165$;
$3.03 \%)$, alopecia $(2 / 165 ; 1.21)$, red face $(3 / 165$; $1.81 \%)$ and varicella zoster virus $(1 / 165 ; 0.6 \%)$. One patient discontinued dupilumab after 4 weeks for generalized lymphadenomegaly.

Topical corticosteroids (TCs) and/or topical tacrolimus and pimecrolimus (TIMs) were used at baseline by $37.57 \%(62 / 165)$ and $23.03 \%$ (38/ 165) patients, respectively. After 16 weeks of treatment, TCs were completely stopped by $27.27 \%(45 / 165)$ of patients, while TIMs were stopped by $6.67 \%(11 / 165)$ of patients. At 52 weeks, $29 / 165(17.57 \%)$ patients referred to occasional use of TCs, while 8/165 (4.85\%) never stop TIMs use.

Our study reported data about efficacy and safety of long-term treatment with dupilumab of moderate-to-severe AD. Actually, in Italy dupilumab represents the only on-label therapeutic option in adult patients with $\mathrm{AD}$ and with contraindications and/or failure of CsA. The efficacy and safety were demonstrated in clinical trials: the identically designed SOLO 1 and 2 studies reported results at 16 weeks of treatment with dupilumab in monotherapy [2];
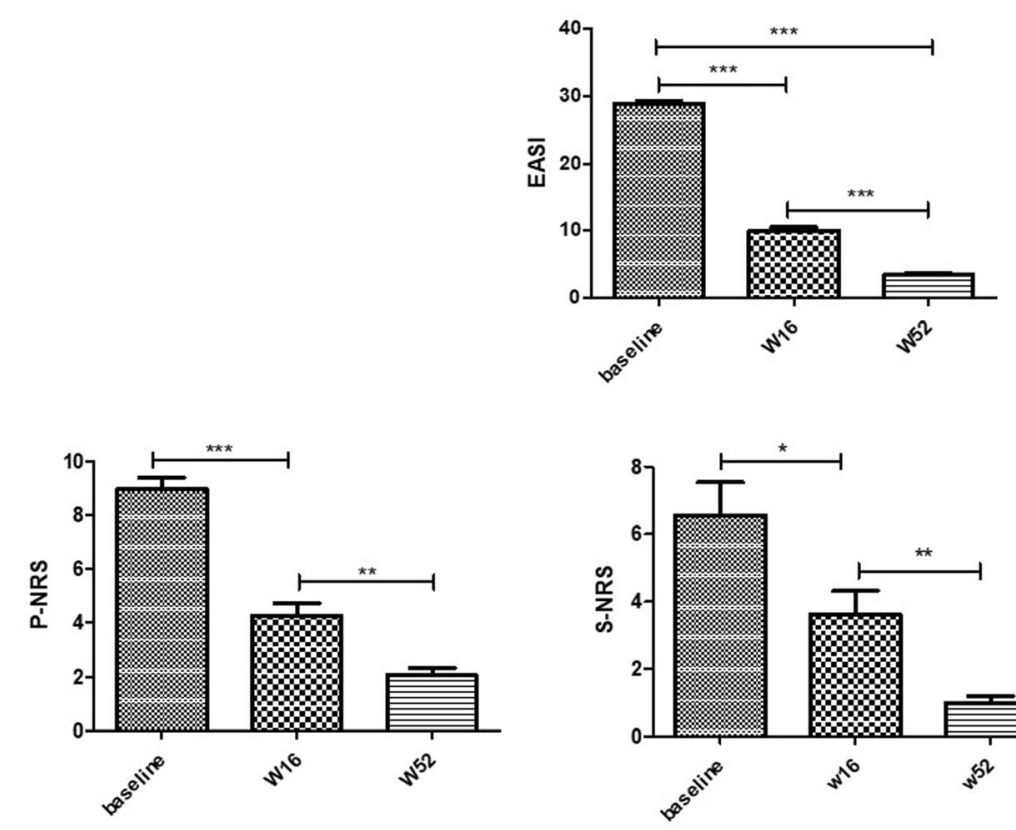

Fig. 1 Improvement in terms of mean variation of EASI, P-NRS, S-NRS and DLQI from baseline to weeks 16 and 52. Mean values of EASI (Eczema Area and Severity Index), S-NRS and P-NRS (Sleep and Pruritus Numerical Rating Score) and DLQI (Dermatology Life quality Index)
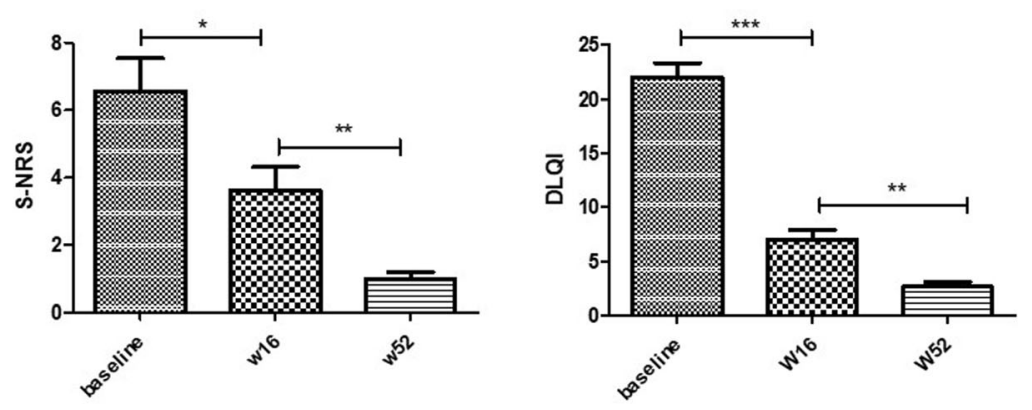

of study population before (week 0, W0) and after 16 weeks (W16) and W52 of dupilumab treatment. Statistical significance was assessed by the Mann-Whitney test and Fisher test: ${ }^{* * *} p<0.0001,{ }^{* *} p<0.001,{ }^{*} p<0.01$ 


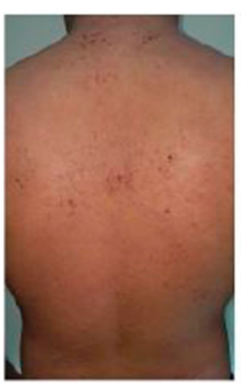

W0

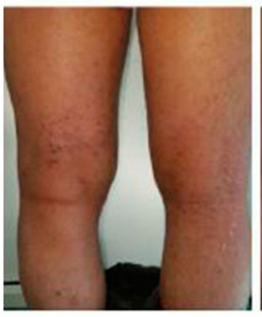

W0

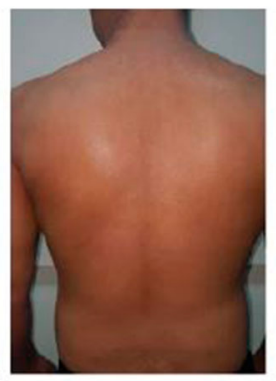

W16

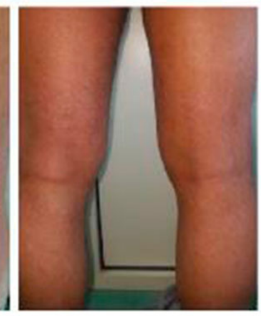

W16

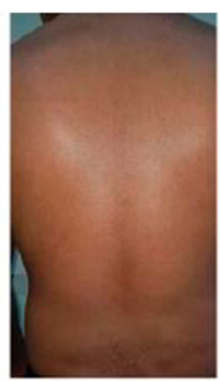

W52

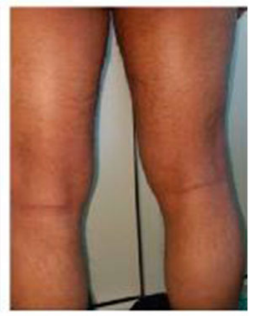

W52
Fig. 2 Improvement of a patient from baseline to 16 and 52 weeks of treatment with dupilumab

while the CAFÉ and CHRONOS studies assessed the drug administered concomitantly with topical corticosteroids, at 16 and 52 weeks of treatment, respectively [3, 4]. Currently, data regarding its daily practice setting, real-word effectiveness and tolerability up to 52 weeks are limited [5-7].

We evaluated 165 adult patients affected by severe $\mathrm{AD}$ and treated with dupilumab; we found that 71/165 (43.04\%) of them had adultonset $\mathrm{AD}$, higher than reported previously $[5,8]$. In our study group, the most frequent clinical phenotype was the flexural dermatitis $(52.19 \%)$, which presented with similar distribution (without significant difference) in both groups, persistent and late-onset. The coexistence of more than one phenotype was found in 25/165 (15.15\%) patients. As reported in other studies, the more frequent associations were flexural dermatitis with head-and-neck eczema and/or hand eczema $[5,8]$. No significant difference between the persistent or adult-onset group was found regarding distribution of other phenotypes as well. Regarding therapy, we found significant improvements in disease severity scores that have been evaluated (EASI,
P-NRS and S-NRS, DLQI) (Fig. 2). As matter of fact, we observed that at 52 weeks $68.48 \%$ of patients achieved EASI75 and $19.39 \%$ of patients EASI90, respectively. These percentages of efficacy at 52 weeks are higher than those reported in clinical trials [4] and in other reallife studies [6, 9]. Indeed, Armario-Hita et al. reported an EASI reduction of $79.3 \%$ in 70 patients at 24 weeks [10]. According to previous studies and finding reported by Kreeshan et al., we did not observe an overall significant decrease of serum IgE or a change in the eosinophil count. As regards the safety of dupilumab, conjunctivitis has proven to be the most frequent $\mathrm{AE}$, reported also by Kreeshan et al. in $16.02 \%$ of patients. Most of our patients had mild-to-moderate conjunctivitis which was treated with lubricants and/or steroids eye drops. In our cohort, new-onset conjunctivitis was observed in $9.69 \%$ of patients, in accordance with the literature. The reported incidence in clinical trials and in real-life studies ranges from $5 \%$ to $28 \%$, and from $6 \%$ to $62 \%$ of dupilumab-treated patients, respectively $[3,4,6,11]$. Regarding other AEs, we observed a lower incidence of red face and no case of arthralgia, reported by Kreeshan et al. in 10 and 6 patients, respectively. The interruption of therapy had occurred for severe conjunctivitis in 2 patients and generalized lymphadenomegaly in one patient. Other possible systemic causes were excluded in the patient who developed lymphadenomegaly. The blood chemistry and instrumental tests carried out were normal. The lymphadenomegaly disappeared after dupilumab was discontinued. Our study found low discontinuation rates of dupilumab treatment (1.8\%). This percentage of discontinuation is lower compared to the multicentre cohort of Ariëns et al. (8.1\%) and retrospective daily practice study of Jo et al. (12\%) $[6,12]$.

As suggest by Kreeshan et al., our data confirm that dupilumab can represent a long-term treatment for moderate-to-severe adult $\mathrm{AD}$, beyond 16 weeks. Furthermore, in our experience, dupilumab demonstrated a favourable safety profile at 52 weeks and only a few patients had to discontinue the treatment because of AEs. Our study has several 
limitations including its retrospective nature and the small sample size.

\section{ACKNOWLEDGEMENTS}

Funding. No funding or sponsorship was received for this study or publication of this article.

Authorship. All named authors meet the International Committee of Medical Journal Editors (ICMJE) criteria for authorship for this article, take responsibility for the integrity of the work as a whole, and have given their approval for this version to be published.

Disclosures. Napolitano M. acted as speaker, consultant and advisory board member for Sanofi, Abbvie, Leo Pharma; Patruno C. acted as investigator, speaker, consultant, and advisory board member for AbbVie, Eli Lilly, Novartis, Pfizer and Sanofi; Gabriella Fabbrocini is a member of the journal's Editorial Board, and has been principal investigator in clinical trials sponsored by and/or and has received personal fees from AbbVie, Abiogen, Almirall, Celgene, Eli Lilly, Leo Pharma, Novartis, Sanofi, and UCB; Maria Ferrillo, Cataldo Patruno, Massimiliano Scalvenzi and Mirella D'Andrea have nothing to disclose.

Compliance with Ethics Guidelines. All procedures performed in studies involving human participants were in accordance with the ethical standards of the institutional and/or national research committee and with the 1964 Helsinki Declaration and its later amendments or comparable ethical standards. The study has been approved by ethics committee of University of Naples Federico II and Informed consent was obtained from all individual participants involved in the study.

Data Availability. The datasets generated during and/or analyzed during the current study are available from the corresponding author on reasonable request.
Open Access. This article is licensed under a Creative Commons Attribution-NonCommercial 4.0 International License, which permits any non-commercial use, sharing, adaptation, distribution and reproduction in any medium or format, as long as you give appropriate credit to the original author(s) and the source, provide a link to the Creative Commons licence, and indicate if changes were made. The images or other third party material in this article are included in the article's Creative Commons licence, unless indicated otherwise in a credit line to the material. If material is not included in the article's Creative Commons licence and your intended use is not permitted by statutory regulation or exceeds the permitted use, you will need to obtain permission directly from the copyright holder. To view a copy of this licence, visit http://creativecommons.org/licenses/by$\mathrm{nc} / 4.0 /$.

\section{REFERENCES}

1. Kreeshan FC, Al-Janabi A, Warren RB, Hunter HJA. Real-world experience and laboratory monitoring of dupilumab in patients with moderate to severe atopic dermatitis in a tertiary centre. Dermatol Ther (Heidelb). 2020. https://doi.org/10.1007/s13555020-00469-6

2. Simpson EL, Bieber T, Guttman-Yassky E, et al. Two phase 3 trials of dupilumab versus placebo in $\mathrm{AD}$. N Engl J Med. 2016;375:2335-48.

3. De Bruin-Weller $\mathrm{M}$, Thaçi $\mathrm{D}$, Smith $\mathrm{CH}$, et al. Dupilumab with concomitant topical corticosteroids in adult patients with $\mathrm{AD}$ who are not adequately controlled with or are intolerant to ciclosporin A, or when this treatment is medically inadvisable: a placebo-controlled, randomized phase 3 clinical trial (LIBERTY AD CAFÉ). Br J Dermatol. 2018;178:1083-101.

4. Blauvelt A, de Bruin-Weller M, Gooderham M, et al. Long-term management of moderate-to-severe AD with dupilumab and concomitant topical corticosteroids (LIBERTY AD CHRONOS): a 1-year, randomised, double-blinded, placebo-controlled phase 3 trial. Lancet. 2017;389:2287-303.

5. Nettis E, Fabbrocini G, Ortoncelli M, et al. Longterm effectiveness of dupilumab up to 52 weeks in atopic dermatitis in 253 adult patients. Br J Dermatol. 2020. https://doi.org/10.1111/bjd.19577. 
6. Ariëns LF, van der Schaft J, Spekhorst LS, et al. Dupilumab shows long-term effectiveness in a large cohort of treatment-refractory atopic dermatitis patients in daily practice: 52 -weeks results from the Dutch BioDay registry. J Am Acad Dermatol. 2020. https://doi.org/10.1016/j.jaad.2020.08.127.

7. Fargnoli MC, Esposito M, Ferrucci S, et al. A 48-week update of a multicentre real-life experience of dupilumab in adult patients with moderate-tosevere atopic dermatitis. J Dermatol Treat. 2020. https://doi.org/10.1080/09546634.2020.1773379.

8. Hello M, Aubert H, Bernier C, Néel A, Barbarot S. Dermatite atopique de l'adulte [Atopic dermatitis of the adult]. Rev Med Interne. 2016;37(2):91-9. https://doi.org/10.1016/j.revmed.2015.10.345 [Article in French].

9. Jo CE, Georgakopoulos JR, Ladda M, et al. Evaluation of long-term efficacy, safety, and reasons for discontinuation of dupilumab for moderate-to-severe atopic dermatitis in clinical practice: a retrospective cohort study. J Am Acad Dermatol. 2020;82:1530-2.

10. Armario-Hita JC, Pereyra-Rodriguez J, Silvestre JF, et al. Treatment of moderate-to-severe atopic dermatitis with dupilumab in real clinical practice: a multicentre, retrospective case series. Br J Dermatol. 2019;181(5):1072-4.

11. Faiz S, Giovannelli J, Podevin C, et al. Groupe de Recherche sur l'Eczéma aTopique (GREAT), France. Effectiveness and safety of dupilumab for the treatment of atopic dermatitis in a real-life French multicenter adult cohort. J Am Acad Dermatol. 2019;81:143-51.

12. Jo CE, Georgakopoulos JR, Ladda M, et al. Evaluation of long-term efficacy, safety, and reasons for discontinuation of dupilumab for moderate to severe atopic dermatitis in clinical practice: a retrospective cohort study. J Am Acad Dermatol. 2020;82(6):1530-2. https://doi.org/10.1016/j.jaad. 2020.02.029. 